\title{
An Efficient Dorsal Hand Vein Recognition Security System using Arduino and GSM Technology
}

\author{
N. Rajalakshmi, Ramsankar. M. P, P. Manimegalai
}

\begin{abstract}
With the onset of maximum power, modest figuring and more prominent unpredictability, biometric verification has turned out to be conceivable at each scale in light of its more secure nature and furthermore easy to use conduct. Compare to other biometrics, vein biometric is a decent verification characteristic among others. The dorsal hand vein recognition is an emerging biometric procedure which is utilized for verification purposes in many applications. In this work preprocessing is done by median filter and region of interest such as veins separated from the muscles and bones through adaptive $K$ means clustering algorithm.The proposed method extracts the dorsal hand vein pattern features by using LBP and Repeated Line Tracking algorithm. Finally recognition and authentication is done using Artificial Neural Network. Arduino and GSM technology is used in this work to set security preference for the particular user.In order to validate the proposed work, a total of 480 images of dorsal hand veins is involved in this work. In a comparison with four existingverification algorithms, the proposed method achieves thehighest accuracy with lowest error rate.
\end{abstract}

\section{Keywords-dorsal hand vein, Repeated Line Tracking,LBP}

\section{INTRODUCTION}

Biometric innovation is a proficient individual verification and distinguishing proof method. Biometric is the term used to imply the field of numerical examination of novel human features.Biometric system have seen a revived pace of development in the worldwide market of security in the course of recent decades, primarily by expanding necessities in open security against fear-based oppressor exercises, modern violations, and digital false. The following are a portion of the security parameters that are related with biometrics.

- Biometrics depends on the uniqueness of human highlights

- Since biometrics is related with individual, it can't be imparted to other people.

- Biometrics properties can't be lost, except if a genuine mishap occurs.

- It can't be duplicated.

As one of the standard branches, vein acknowledgment has drawn much consideration among analysts and assorted clients. Anatomically, veins are blood conveying vessels interlaced with muscles and bones, and the key capacity of

Revised Manuscript Received on 14 August, 2019.

N.Rajalakshmi, Associate Professor, Dept of BME, KAHE, Coimbatore, Tamilnadu, India. ( Email: praniraji1@gmail.com)

Ramsankar.M.P,UG student, Dept of ECE, KAHE, Coimbatore, Tamilnadu, India. ( Email: ramsankar7@icloud.com)

P.Manimegalai, Assoc.Professor, Dept of BME, Karunya Institute of TechnologyandSciences,Coimbatore, Tamilnadu, India. ( Email: manimegalai.vairavan@gmail.com) the vascular framework is to supply oxygen to each piece of the body. The spatial course of action of vascular system in the human body is steady and vein examples of people are unique, even between indistinguishable twins. Biometrics is the investigation of distinguishing an individual utilizing its conduct and physiological highlights. In this paper, vein of the back of the hand is concentrated because it is particularly obvious, simple to obtain, and effective to process. As contrasted and other mainstream biometric characteristics, for example, face or unique mark, the hand vein has a few recognized benefits. Relatively, vein design based biometric frameworks have the accompanying preferences.

Even though vein recognition system have lot of benefits ,still there are a few difficultiesshould have been dealt in future in order to get efficient Dorsal vein biometric recognizable frameworks. To start with, poor lighting at the catching gadget may make the picture show up amazingly dull or brilliant. Furthermore, in Dorsal vein obtaining process, the situation of the back of the hand and the camera is close, which causes optical obscuring on the caught picture and in the event that the hand isn't guided with a legitimate hand-docking outline, at that point a slight misalignment will be there, which may diminish the acknowledgment rate in the end making the coordinating procedure be erroneous. Third actuality is, every unique individual has diverse pressure levels when keeping the turn in a fisted position, bringing about differed size of palm dorsal surface. The subsequent commotion must be expelled however much as could be expected and conventional dorsal-vein acknowledgment techniques utilize some startling picture preparing calculations to beat the issues referenced.

\section{PREVIOUS WORK}

N.A. khan, et al., [1]proposed a more secure system of dorsal hand vein identification which is led to a rise in developing biometric systems. Dorsal hand vein patterns are an emerging biometric which has attracted the attention of many researchers. Different approaches have been used to extract the vein pattern and match them.Naushad Ali, et al., [2] confirmed in his work that his aim was at reducing the dimension of the training set by building an adaptive estimating equation or a quadratic inference function that 
combines the co-variance matrix and the vectors in the training set, here the phases applied on the hand dorsal vein pattern, feature extraction using quadratic inference is present.Maelikamamode khan, et al., [3] presented a cartesian based co-ordinate system (CBM) to represent dorsal and vein pattern and also introduce a novel method to extract the dorsal hand vein pattern that is based on the generalized method. However, this method leads to high dimensional covariance matrix which is singular or rank deficient. To overcome this problem, they use a fast and accurate algorithm to compute the inverse using the MorrePenrose method. The biometric system developed is tested on a database of 100 images. The False Acceptance Rate (FAR), False Rejection Rate (FRR) were computed.R.K.Subramanian et al.,[4] stated that there is a distinctive pattern of veins at the back of the hand. When a fist shape is made by the hand, a unique pattern of blood vessel is found. This pattern can be used a biometric security systems. The postion of the hand while capturing the pattern and the effect of mental, physical and biological changes, aging in the human being concerned are the major problems that were addressed. They studied one of the main challenges viz., the position of the hand while capturing the main pattern and discuss a suitable method of implementing the same. The paper also outlines a discussion on the other challenges.JayanthiYusmah sari, et al., [5] commented biometric system is a more promising identification method than using cards and passwords.one of the physiological biometrics that is currently being developed is the dorsal hand vein pattern. The pattern of vein contains information that is able to authenticate individual identities for the purpose of personal authentication automatically. Vein patterns can be recognized by using a feature extraction approach.Heenayae, et al.,[6] justifies that no biometric system provides $0 \%$ false acceptance rate and $0 \%$ false rejection rate. In order to prove which method is efficient in identifying dorsal hand vein images different techniques are explored in this work. LPP which is a non-linear method is being applied on dorsal hand vein features. This technique presents the extracted features into a reduced dimensional space and is compared with pixel by pixel method. FAR and FRR are recorded for both methods and was found LPP proves to be a more efficient method by having less FAR and FRR. Pooja, et al.,[7] briefed different feature extraction methods. In this work, three feature extraction and representation techniques namely Hough lines transform, Pixel by Pixel method and Directional coding method have been explored and implemented. These techniques are applied on 500 images obtained from 100 individuals of different age. Mahalanobis Distance Correlation Percentage has been used for classification in this work. From the experimental results, it was concluded that Pixel by Pixel method proved to be the best feature extraction technique with a False Rejection Rate (FRR) of $0.03 \%$.Krishna, et al.,[8]discussed classification of biometrics.In his work he analysed various physical and behavioural biometrics.

\section{III.PROPOSED METHOD}

In the proposed method we discuss about different image processing strategies. Initially filtering process is applied on the captured vein pattern image to remove thenoise from the image.

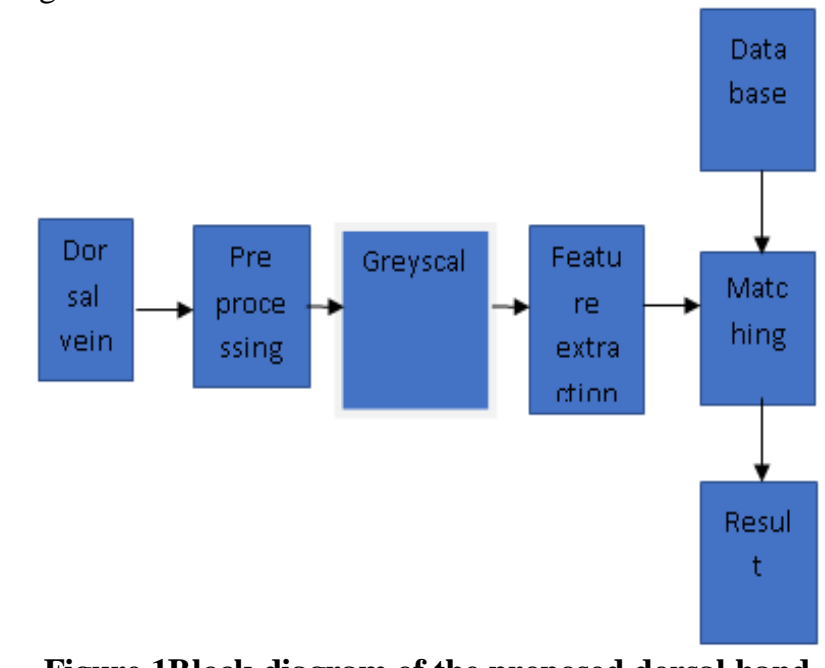

Figure 1Block diagram of the proposed dorsal hand vein recognition system

\subsection{Preprocessing}

In this work a total of dorsal hand veins480 images of 103 individuals obtained fromweb such as University of Las Palamas de Gran Canaria, Spain. Some of the images obtained from obtained from trustable online Database.There are two major steps in thepreprocessing stage, which are image resizing[9] and ROI extraction,preprocessing is achieved through median filter.ROI extraction is carried out by adaptive $\mathrm{k}$ means clustering.

\subsection{Dorsal Hand Vein Feature ExtractionutilizingLbp}

The Local binary pattern (LBP) operator isa texture descriptor based on the gray levelcomparison of a neighborhood of pixels. Theoriginal operator considers a $3 \times 3$ neighborhood of 8 pixels around a center pixel[12]. This neighborhood isthresholded by the value of the center pixel and theresult considered as a binary number or its decimal equivalent [10]. LBP operator as defined inEquation (1).

$$
\begin{aligned}
& L B P_{P, R}(I c)=\sum_{p=1}^{p} s\left(z_{p}-z_{c}\right) 2^{p-1}(3) \\
& \mathrm{s}(\mathrm{u})=1 \mathrm{ifu} \geq 0
\end{aligned}
$$$$
0 \text { otherwise }
$$

LBP operator is based on gray level differences in local neighborhoods. Therefore it has the ability to extract discriminative features from dorsal vein images.In this work dorsal vein image of size $256 \times 256$ is taken and parameters assigned are $\mathrm{P}=16, \mathrm{R}=7$ applied on 18 sub images of size $49 \times 49$ with vein width of about $2-15$ pixels.

\section{RECOGNITION BASED ON ARTIFICIAL NEURAL NETWORK}

Among the different customary methodologies of sample acknowledgment, the measurable methodology has been most seriously examined. After the feature extraction the next step is to feed through the image to artificial neural network for classification. The recognition is done by studying the features of the image. 


\subsection{Results And Discussions}

The proposed method was implemented inMatlab.After the classification of doralvein performance metric like FAR,FRR,accuracy are computed for this work against existing work.As shown in Table 1, the highest recognition result obtained by proposed method with $99.12 \%$ of accuracy while the lowest error than existing methods. The performance graph of FAR, FRR, accuracy and EER is plotted in Figures 2 to4.Figure 6-14 shows output from each and every stages of recognition system.

\begin{tabular}{|l|l|l|l|l|l|}
\hline $\begin{array}{l}\text { S.N } \\
\text { o }\end{array}$ & $\begin{array}{l}\text { Techniq } \\
\text { ues }\end{array}$ & FAR & FRR & $\begin{array}{l}\text { ACCUR } \\
\text { ACY }\end{array}$ & EER \\
\hline 1 & Proposed & $\begin{array}{l}8.981 \\
7\end{array}$ & $\begin{array}{l}0.932 \\
45\end{array}$ & 99.12 & 0.71 \\
\hline 2 & Lin et al & $\begin{array}{l}8.451 \\
2\end{array}$ & $\begin{array}{l}0.921 \\
46\end{array}$ & 98.31 & 1.77 \\
\hline 3 & $\begin{array}{l}\text { Wang et } \\
\text { al }\end{array}$ & $\begin{array}{l}7.452 \\
82\end{array}$ & $\begin{array}{l}0.821 \\
06\end{array}$ & 93.87 & 5.88 \\
\hline 4 & $\begin{array}{l}\text { Kumar et } \\
\text { al }\end{array}$ & $\begin{array}{l}7.852 \\
13\end{array}$ & $\begin{array}{l}0.881 \\
23\end{array}$ & 95.64 & 4.19 \\
\hline
\end{tabular}

Table 1: Comparative analysis of proposed work on dorsal hand vein based biometric recognition with other existing techniques

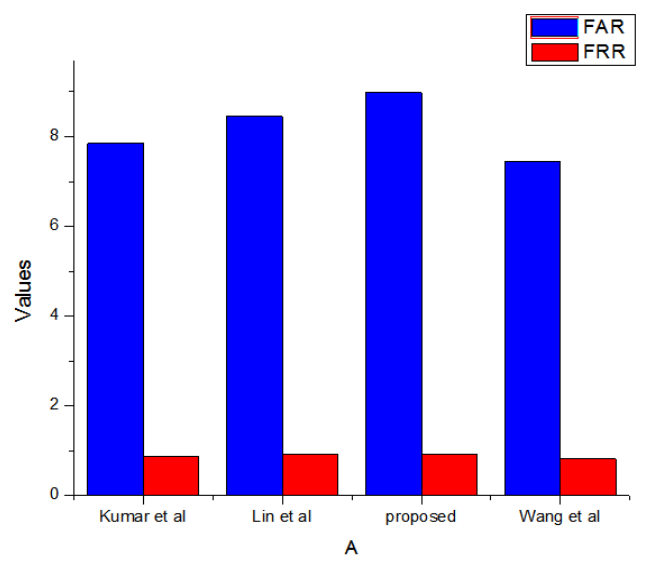

Figure 2: Comparison ofFAR,FRR of different dorsal hand vein recognition system

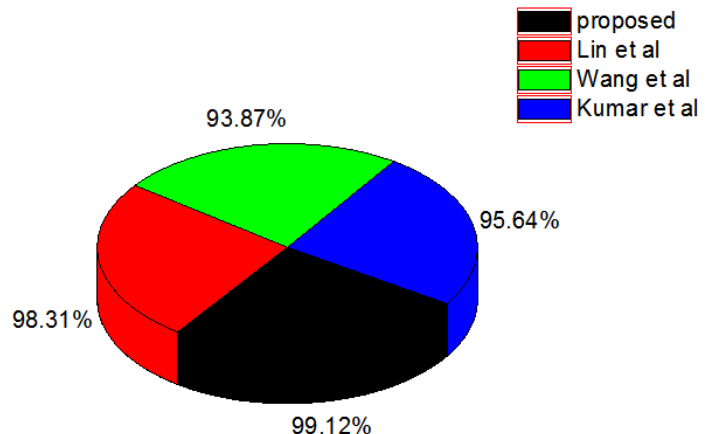

Figure 3: Comparison ofaccuracy of different dorsal hand vein recognition system

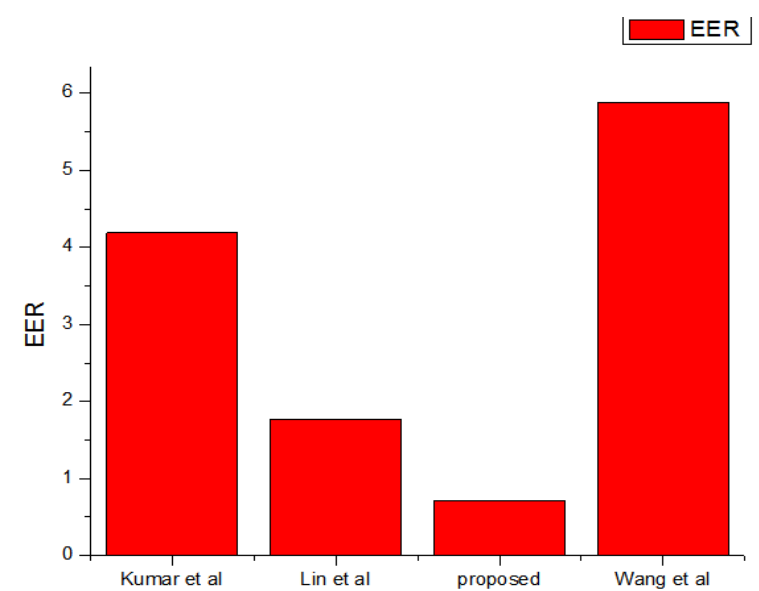

Figure 4: Comparison ofEER of different dorsal hand vein recognition system

After getting the output it is validated and hardware part is implemented through arudino and GSM technology. When a validated passage is made the security opens up after every single coordinating methodology are finished. On the off chance that an unauthenticated section is made, at that point the venture framework would make a signal commotion and sends a Text message to the approved versatile number through GSM.
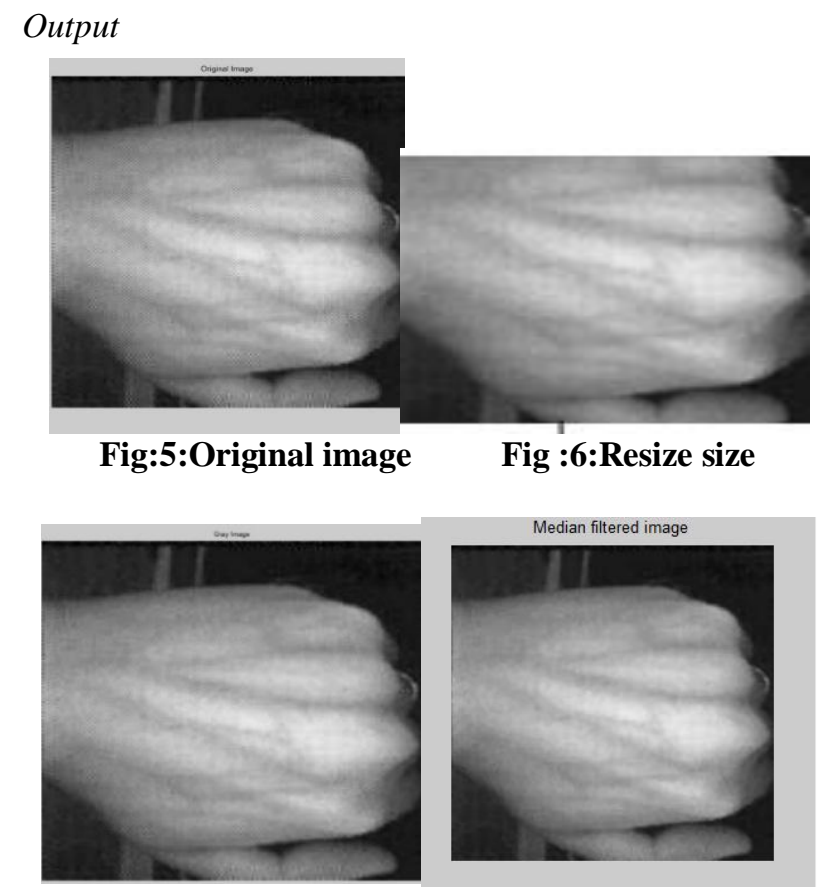

Fig:7:Grey image Fig:8:Median filter image

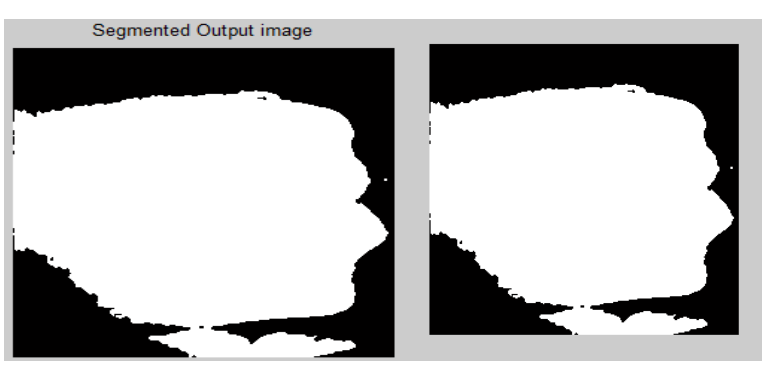

Fig:9:Segmented output image 

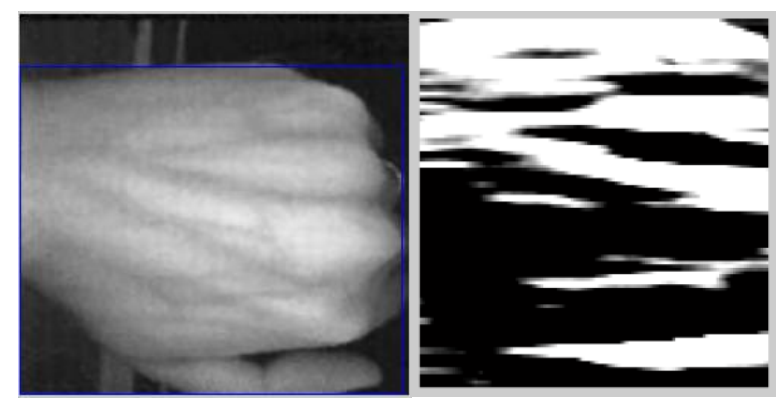

Fig 10:Blue line extraction Fig 11: Extraction of biometric features
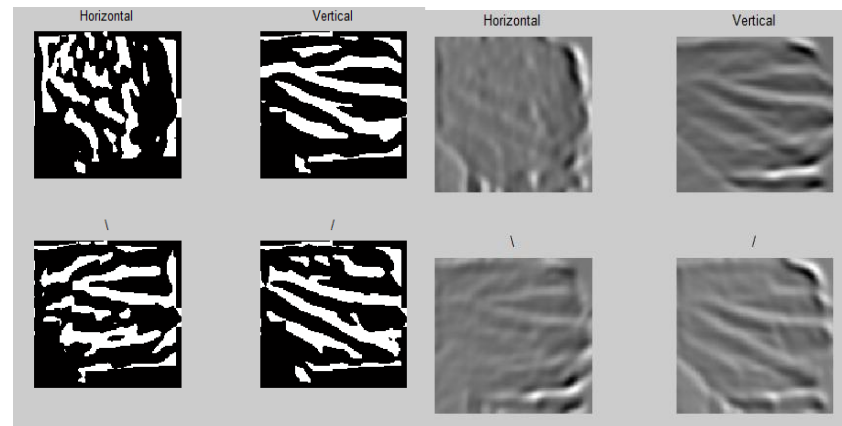

Fig:12 second order derivatives and biometric fusion patterns

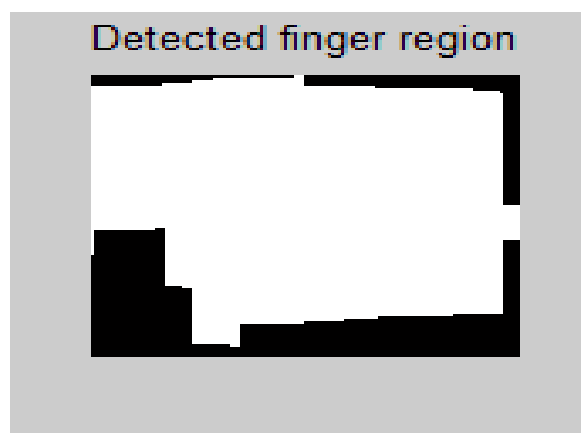

Fig:13:Finger region detection

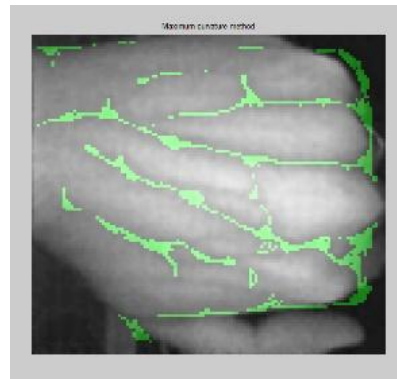

(i)

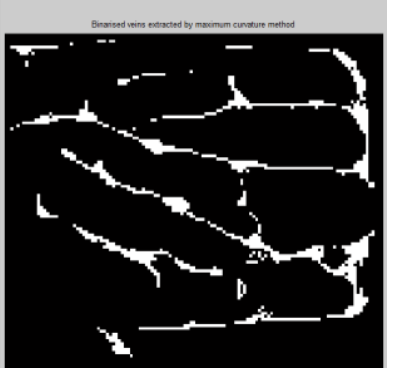

(ii)

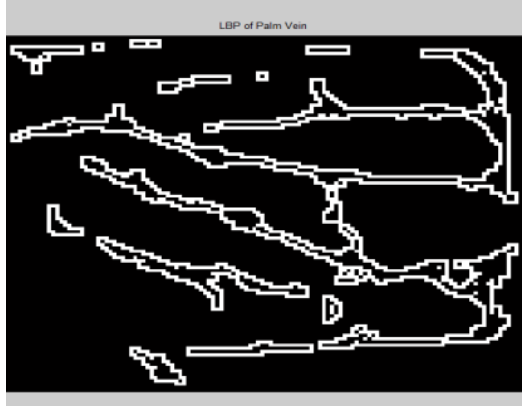

(iii)
Figure 14i) Binarised Vein Ii)Maximum Curvature Iii) LBP Of Dorsal Hand Vein

\section{CONCLUSION}

In this work, a dorsal hand vein biometric recognition was presented, The proposed system is evaluated using the parameters of accuracy, FAR and FRR. The experimental results show improvedperformance of the system, when compared with other current techniques, indicating the effectiveness of our proposed technique. This technique provides the highest accuracy of $99.1 \%$ and minimum EER. With the resulting authentication through matching result, the pre-stored authenticated user data is referred and an Arduino based SMS alert function is used to inform the authenticity of the entering user to the registered phone number. Thearduino is used for controlling the buzzer and the GSM, it has a pre-defined program through which the entire operation rely upon. After finding the authenticity of the user, the pop-up blog is recognised by the arduino program and sends the SMS alert through GSM module, to the user defined in the program. This results in a fullyfunctioning efficient dorsal hand vein recognition security system using Arduino and GSM technology

\section{REFERENCES}

1. N.A. Khan, R.K. Subramanian,Lowdimentional representation of dorsal hand vein features using principle component analysis, International journal of computer, electrical, automation control and information engineering, Vol:3,No:1,2009.

2. Naushad Ali Mamode,(IJCSIS) International journal of computer science and Information security,Vol:6,No:3,2009.

3. MaelikaHeenayae Khan and Naushad Ali Mamode, A new method to extract dorsal hand vein pattern using quadratic inference function,(IJCSIS) International Journal of Computer science and Network security, Vol:9,No:12,Dec-2009.

4. R.K. Subramanian, MaelikaMehrNigar M. Heenayae, An efficient dimension reduction algorithm to extract dorsal hand vein pattern based on generalized method of moments and moore-pensore generalized inverse procedure, University of Mauritius Reasearch journal, Vol:15,2009

5. JayantiYusmahSari, Multispectral dorsal hand vein recognition based on local line binary pattern, journal of science and information,11/2,PP 95-102,2018

6. M.Heenayae,Mamod.N.Khan, A novel technique to represent dorsal hand vein features using LPP, University of Mauritius Research journal,Vol:17,2011.

7. B Nagaraj., andVijayakumar, "Tuning of a PID controller using soft computing methodologies applied to moisture control in paper machine." Intelligent Automation \& Soft Computing 18, no. 4 (2012): 399 411.

8. V.KrishnaSree,P.SudakarRao, Dorsal hand vein pattern authentication by houghpeaks, International Research in Engineering and Technology(IJRET),Vol:03,May-2014.

9. C.Narmatha,P.manimegalai,S.Manimurugan,Journal of medical imaging and health informatics,A lossless compression scheme for grayscale medical images using a P2-Bit short technique,vol.7,1-9,2017.

10. Rajalakshmi.N,lakshmiprabha.V,Segregation of MRI images using hybrid evolutionary clustering algorithm,International journal of biomedical engineering and technology,2014.

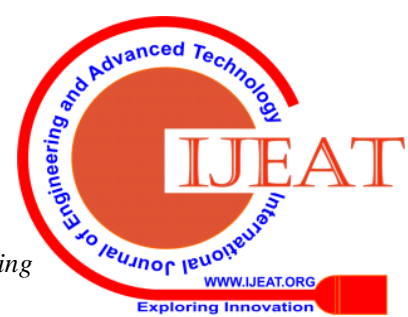


11. Naoto Miura, Akio Nagasaka, TakafumiMiyatake, Feature extraction of finger-vein patterns based on repeated line tracking and its application to personal identification.MachineVision and Applications (2004) 15: 194-203.

12. NatrajanRajalakshmi and Viswanathan Lakshmi Prabha, MRI brain image classification-a hybrid ap[porach,International journal of imaging system and technology,Vol 25,PP-226-244,Sep2015 